\title{
Pregnancy Rate After Fresh Embryo Transfer Versus Vitrified- Thawed Embryo Transfer Cycles: Minia University Experience
}

\section{Original Article}

\author{
Mahmoud H. Ibrahim, Reham Elkhateeb, Ahmad Mahran, Hany H Kamel \\ Infertility and IVF unit, Maternity Hospital, Minia University, Minia, Egypt, Department of \\ Obstetrics and Gynecology, Maternity Hospital, Minia University
}

\begin{abstract}
Objective: To study pregnancy rates after vitrified-thawed embryo transfer and fresh embryo transfer.

Design: Retrospective study.

Setting: Infertility and IVF unit, Minia University Hospital.

Patients and Methods: All women undergone ICSI program during the period from first of January 2010 to December 2014, pregnancy rate was compared between two groups, first group (Group I) had fresh embryo transfer, the second group (Group II) had vitrified -thawed embryo transfer .

Main outcome: Clinical pregnancy rate.

Results: There was insignificant difference between two groups regarding chemical pregnancy rate it was 228 (43\%) in (Group I), 36\% (186) in the second group (Group II). Clinical pregnancy rate $24.5 \%$ (130) versus 22.1\% (114). Implantation rate $28.6 \%$ versus $24 \%$. The rate of single pregnancy was higher in the first group $80 \%$ than in the second group $76.4 \%$. The rate of twins was higher in the second group $23.6 \%$.

Conclusion: A program of vitrified-thawed embryo transfer should be adopted by all IVF center especially in low-income countries to maximize the benefit of the single treatment cycle, increase cumulative pregnancy rate and decrease the cost of repeated cycle stimulation, as the pregnancy rate is almost equal in FET and fresh embryo transfer.
\end{abstract}

Key Words: IVF, vitrified-thawed embryo, and fresh embryo transfer.

Revised: 29 October 2016, Accepted: 1 February 2017

Corresponding Author: Mahmoud H. Ibrahim, MD Faculty of Medicine, Minia University, 61111 Minia, Egypt., E-mail: hosnimahmoud60@yahoo.com, Tel: 01005389725

ISSN: 2090-7265, May 2017, Vol. 7, No. 2

\section{INTRODUCTION}

The use of assisted reproductive technology (ART) is increasing in the world. The rate, efficacy, and safety of this technology are very different among countries. There is an increase in the use of intracytoplasmic sperm injection (ICSI), single fresh embryo transfer (ET) and frozen-thawed embryo transfer (FET) ${ }^{1}$.

In the past decades, in-vitro fertilization and embryo transfer (IVF-ET) has become the most effective treatment for infertility. Acquiring highquality embryos and implanting them successfully in a receptive endometrium are the most important factors for improving the success rate of IVF-ET ${ }^{2}$.

FET provides several benefits in ART such as increasing cumulative pregnancy rate, decreasing the risk of multiple pregnancy by increasing the use of SET and it also reduces hyperstimulation syndrome ${ }^{3}$. Some description that women who use cryopreservation have a better prognosis, with good ovarian reserve ${ }^{4}$.
In fresh cycles, the endometrium is artificially primed and the embryos could be cryopreserved and used in next cycles when the detrimental effects of high dose of hormones during controlled ovarian hyperstimulation $(\mathrm{COH})$ are disappeared. Identically, in frozen-thawed embryo transfers, endometrial priming may be achieved with the use of estrogen and progesterone, and endometrial growth can be controlled more exactly in $\mathrm{COH}$ cycles 5 .

A recent study revealed safety and cost-effectiveness of FET compared with fresh $\mathrm{ET}^{6}$.

Embryos can be cryopreserved at any stage, from zygote to blastocyst and remain viable for at least several years ${ }^{7}$. Embryo cryopreservation at the pronuclear, cleavage and blastocyst stages has been allowed for multiple transfer cycles from single oocyte retrieval. As the transfer of cryopreserved embryos is less expensive than a second fresh cycle, fertility treatment costs can be optimized $^{8}$. 
No increase in the incidence of prematurity, low birth weight (LBW) and neonatal death were found in the FET group compared with the fresh ET group?.

There is no increase in the incidence of major malformation rates in children born after FET or fresh ET compared with children born after spontaneous conception $^{10}$

\section{PATIENTS AND METHODS}

This retrospective analytic study recruited 1044 ICSI ET cycles (530 fresh embryo-transfer and 514 FET). Upon approval of the ethical committee, the current research was conducted at Maternity Hospital IVF unit, Minia University in the period from January, 2010 to December 2014. All women's files undergone ICSI were studied retrospectively, the selected women considering the condition of the transferred embryo were divided into two groups; the first group (GroupI) included 530 women who had fresh embryo transfer and the second group (Group II) included 514 patients who had frozen-thawed embryo transfer both groups fulfilled the inclusion criteria.

\section{Inclusion criteria:}

- $\quad$ Age must be less than 40 years.

- Normal baseline hormonal profile (FSH, LH, E2, Prolactin and $\mathrm{AMH}$ ).

- Normal uterine cavity confirmed by hysterosalpingogram or hysteroscopy.

\section{Exclusion criteria}

- Women did not fulfill inclusion criteria.

- Women had endocrine or metabolic disorder.

- Women had a history of chemotherapy.

- Women had uterine anomalies, or focal endometrial lesions (fibroid, polyp, adhesion, endometritis..... etc).

- Women with history endometriosis.

- Women with previous ICSI trail.

In group I, controlled ovarian hyperstimulation was achieved by one of three protocols; mid luteal long agonist protocol, short antagonist protocol or a microdose flare protocol (initiation of GnRH agonist on Days 2-3 of the cycle after oral contraceptive-induced withdrawal bleeding and gonadotrophin on the third day of the GnRH agonist $)^{11}$.
Ovum pick up was decided when more than three follicles measuring $17 \mathrm{~mm}$ or more. It was done $34-36$ hours after B-hCG injection, under GA with ultrasound guide.

Embryo transfer day of embryo transfer was determined based on the quantity and quality of embryos either on Day 3 (cleavage embryos) or 5 (blastocysts). Embryo transfer was performed under ultrasound guidance and a serum beta-HCG pregnancy test was performed 16 days after collection.

Blastocyst grading was performed by two trained embryologists. After embryo transfer, excess blastocysts of $3 \mathrm{BB}$ or higher or D3 embryo were frozen using the verification method. Embryo transfer was decided when there were more than one high-quality embryos available, transvaginal ultrasound guided using double lumen needle.

After egg collection, luteal support started by progestogen rectal suppositories $400 \mathrm{mg}$ twice daily (prontogest $400 \mathrm{mg}$ ) and continued until the day of pregnancy test if positive pregnancy test it continued until 14 weeks of pregnancy.

\section{Group II}

This included 514 patients who undergone frozenthawed embryo transfer.

The FET was performed after treatment of the recipients with $17 \beta$-estradiol starting on Day 2 or 3 of the menstrual cycle for at least 15 days before embryo transfer until the (trilaminar) endometrial thickness reached at least $8 \mathrm{~mm}$ (measured by TVUS), Embryos were accepted for transfer if they retained $\geq 50 \%$ of blastomeres intact after thawing. Endometrial thickness was measured on day 7 of the cycle and day 12 . There is a lack of evidence to recommend any one particular protocol in endometrial priming regarding ART outcomes in $\mathrm{FET}^{12}$.

Luteal phase support was done same as in the first group.

Patients had a quantitative serum $\beta$-hCG level performed 11 days after embryo transfer (blastocyst) or 13 days after Day 3 (cleavage embryo) transfers. A pregnancy test was defined as positive if serum $\beta$-hCG levels were $\geq 10 \mathrm{mIU} / \mathrm{ml}$ using the Siemens Immulite 2000 assay. Sensitivity of the assay was $0.4 \mathrm{mIU} / \mathrm{ml}$, with $<1 \%$ cross-reactivity. Intra- and inter-assay coefficients of variability were $<6.6$ and $7.4 \%$, respectively. 


\section{Definition of outcomes}

Successful pregnancy was confirmed by detecting an increased serum $\beta$-hCG concentration 11-13 days after ET. Clinical pregnancy was defined by the observation of a gestational sac with or without a fetal heart beat on ultrasound evaluation on the $35^{\text {th }}$ day after oocyte retrieval.

Implantation rate was defined as the percentage of transferred embryos which results in sonographically visible gestational sacs.

\section{Statistical methods}

Data entry and analysis were all done using SPSS for windows version 13

Quantitative data were presented by mean and standard deviation, while qualitative data were presented by frequency distribution. Chi square test was used to compare between more than one proportion. A statistically significant level was considered when p-value was less than 0.05 .

\section{RESULTS}

The data of 1044 women, who were subjected to ICSI program were recorded retrospectively and analyzed statistically. They were divided into two groups according to the embryo transferred either fresh (group I) or frozen -thawed embryos (group II).
Analysis of patients' characteristics revealed no statistically significant difference in both groups as regard patient characteristics or baseline hormonal profile shown in Table 1

The two groups were compared as regarding different outcomes (Table 2).

In group I, the chemical pregnancy rate was $43 \%$ versus $36 \%$ in group-II. The different was statistically non-significant with a $P$-value of $0.8 . p$ Value 0.8 ).

The clinical pregnancy rates were $24.5 \%$ (130 cases) versus $22.1 \%$ (114) in group-I and II, respectively. Besides, the implantation rate was $28.6 \%$ versus $24 \%$ in group-I and II, respectively.

The rate of single pregnancy was higher in the first group $(80 \%)$ than in the second group $(76.4 \%)$. The rate of twins was higher in group-II $(23.6 \%)$ than in group-I $(11.6 \%)$. The rate of triplets was $8.4 \%$ in the first group while no triplet was reported in the second group.

Also, there were no statistical differences between 2 groups regarding abortion, ectopic or term pregnancy. As regard mode of delivery, there were 9 cases NVD representing $8.3 \%$ in group-I compared to 13 cases in group II $(13.6 \%)$ with no statistical difference.

Table 1: Patient characteristics and baseline hormonal profile of both groups.

\begin{tabular}{|c|c|c|c|c|c|}
\hline Characters of the women & \multicolumn{2}{|c|}{ Group I(n=530) } & Group II(n=514) & & $P$ value \\
\hline $\begin{array}{l}\text { Age of the women } \\
\text { (years) }\end{array}$ & $5 \pm 30.1$ & & $4.8 \pm 27.5$ & & 0.1 \\
\hline Husband age (years) & $9.8 \pm 36.2$ & & $12.2 \pm 38.1$ & & 0.2 \\
\hline Parity of the women & $1.6 \pm 0.8$ & & $1.3 \pm 0.8$ & & 1 \\
\hline Type of infertility & $\mathrm{N}$ & $\%$ & $\mathrm{~N}$ & $\%$ & $\mathrm{X}$ \\
\hline Primary & 272 & 51.3 & 250 & 48.6 & \\
\hline Secondary & 258 & $48.7 \%$ & 264 & 51.4 & 0.6 \\
\hline FSH (IU/L) & $5.2 \pm 2.5$ & & $4.9 \pm 2.7$ & & 0.4 \\
\hline LH (IU/L) & $6.2 \pm 3.6$ & & $7.5 \pm 5.6$ & & 0.5 \\
\hline Serum Prolactin (MIU/L) & $7.9 \pm 2.1$ & & $15.4 \pm 8.1$ & & 0.3 \\
\hline AMH (ng/ML) & $4.9 \pm 2$ & & $5.0 \pm 2.1$ & & 0.8 \\
\hline E2 (pmol/l) & $65.2 \pm 8.8$ & & $48.8 \pm 4.1$ & & 0.1 \\
\hline
\end{tabular}


Table 2: Different outcomes parameters of both groups.

\begin{tabular}{lccc}
\hline & $\begin{array}{c}\text { Group } \\
\text { 1n=530 }\end{array}$ & $\begin{array}{c}\text { Group } 2 \\
\mathrm{n}=514\end{array}$ & P-VALUE \\
\hline $\begin{array}{l}\text { Chemical } \\
\text { Pregnancy Rate }\end{array}$ & 0.8 & $(\% 36) 186$ & $(\% 43) 228$ \\
$\begin{array}{l}\text { Implantation rate } \\
\text { Clinical Pregnancy }\end{array}$ & 0.2 & $123(24 \%)$ & $152(28.6 \%)$ \\
Rate & 0.1 & $114(\% 22.1)$ & $130(\% 24.5)$ \\
Single & 0.3 & $87(76(\%)$ & $(10480 \%)$ \\
2 Sacs & 0.04 & $(\% 23.6) 27$ & $(\% 11.6) 15$ \\
More than 2 & 0.01 & 0 & $11(8.4 \%)$ \\
Pregnancy outcome :- & & & \\
Abortion & 0.02 & $15(13.1 \%)$ & $24(18.3 \%)$ \\
Ectopic & 1 & $3(1.9 \%)$ & $2(1.7 \%)$ \\
Full-term & 0.93 & $96(84.6 \%)$ & $104(80 \%)$ \\
NVD & 0.82 & $13(13.6 \%)$ & $9(8.3 \%)$ \\
ELSCS & 0.64 & $83(86.4 \%)$ & $95(91.7 \%)$ \\
\hline NVD normal vaginal delivery & ELSCS & lower segment cesarean section
\end{tabular}

\section{DISCUSSION}

IVF-ET has now been applied for thirty years and the clinical pregnancy rate has increased steadily with the development of advanced embryo culture systems and improved understanding of the endocrinology of $\mathrm{COH}$. It is known that high-quality embryos, endometrial receptivity and the need for synchrony between embryo development and endometrial growth are the three most important factors affecting the successful establishment of pregnancy ${ }^{13}$ so the need for cryopreservation become mandatory in IVF centers,

Effective techniques for cryopreservation of surplus embryos at all developmental stages are therefore imperative to maximize the cumulative pregnancy rates between fresh and frozen embryos from single cycle oocyte retrieval. The conventional slow freezing is the classic standard method of cryopreservation. However, vitrification is a promising new technology for embryo cryopreservation adopted by IVF centers around the world $^{14}$.

In the present study, the clinical pregnancy rates were $24.5 \%$ and $22.1 \%$, in group I and II, respectively with no significant difference, consistent with the obtained results by Fitzmaurice et $a l^{15}$ and Check et $a l^{16}$. On the other hand, While Zhu et al., $2011^{17}$ showed higher pregnancy rates in frozen blastocysts than in fresh transfer cycles. The clinical pregnancy rate of fresh and frozen blastocyst transfer groups were $36.4 \%$ and $55.1 \%$, respectively $(p<0.05)$. Also, Feng et al., 200913 found that clinical pregnancy rate in frozen -thawed embryo transfer was significantly higher than in fresh embryo transfer $(63.1 \%$ vs. $47.0 \%$, respectively; $p$-value $<0.01$ ). Noteworthy, it was suggested that high estradiol concentrations influence endometrial receptivity and synchrony between the embryo and the endometrium in patients with high ovarian response during the fresh embryo transfer. Thus frozen cycles had higher pregnancy rate because of lower levels of serum estradiol.

In the current study, the chemical pregnancy rate was higher in the fresh embryo transfer than in frozen-thawed embryo transfer, $43 \%$ versus $36 \%$, respectively. Basirat et al., 2016 (1) documented non-significant difference of the biochemical pregnancy rate $(23 \%$ in FET versus $18.8 \%$ in fresh embryo transfer). Alike, Aflatoonian et al., (3) reported that biochemical pregnancy rate was $27 \%$ (54200/) in the FET group versus $22.1 \%(122500 /)$ in the fresh embryo transfer group.Also, Check et al ${ }^{16}$ obtained a significantly higher pregnancy rate in frozen-thawed embryo than fresh embryo transfer in recipients patients (63.4 versus $43.6 \%$ ) attributed to the absence of high serum Estradiol in the recipients patient.

In the present research, the implantation rate was higher in fresh embryo transfer group than frozen embryo transfer group (28.6\% versus $24 \%$ respectively), but was statistically non-significant with a $P$-Value of 0.2). Zhu et al $2011^{17}$ showed implantation rates in frozen blastocysts was higher than in fresh transfer cycles. The implantation rate of the fresh and frozen group was $25.2 \%$ and $37.0 \%(p<0.05)^{26}$. They explained theses results by distinct endometrial receptivity and better symmetry between the embryo and endometrial development in frozen embryo cycles.

It is important to consider in the fresh embryo transfer cycles we select the best fresh embryos for transfer. Thus, the residual embryos that survive the freezing and thawing methods supposedly have a reduced fortune of implantation. However, the implantation and fertility rates did not diminish in the frozen group when compared with the fresh embryo transfer group in this study. To illustrate the similar results in the current study between the fresh and frozen embryo transfers, multiple factors may be considered. Recent development in methods of verification that ensure more survival and better possible development after thawing ${ }^{18}$.

In the current study, the rate of multiple pregnancy was higher in FET $(23.1 \%)$ than in fresh embryo $(20 \%)$, consistent with the obtained by Feng et $a l^{(13)}$, whereas the multiple pregnancy rate was significantly higher in frozen embryo transfer than in fresh embryo transfer $(46.9 \%$ vs $28.8 \%$ ). Also, they reported no significant differences in the miscarriage rate between the two groups $(11.5 \%$ vs. $8.0 \%$ ) comparable to our study rates of miscarriage that were $18.3 \%$ and $13.1 \%$ in group-1 and II, respectively. Similar results were also revealed in the research by Belva et al., $2008^{9}$. 
On the contrary to our results, Aflatoonian et al. ${ }^{(3)}$ found that spontaneous abortion rate in FET group was significantly higher than in fresh ET group .

In two-thirds of the implantation failures, the primarily responsible source of failure is the impairment of the ER, whereas the embryo itself is responsible for only one-third of the failures ${ }^{19}$, It has been reported that embryos that have better cleaved during the post- thaw time have the significantly higher fortune of implantation and a great number of uncleaved frozen embryos have chromosomal aberrations ${ }^{20}$.

Basirat et al, 2016(1) concluded that there is no significant difference between the fresh ET and the FET cycles, however, the embryos are able to be stored for subsequent ART. Therefore, we recommend FET cycles as an option alongside the fresh ET.

\section{CONCLUSION}

In conclusion, no statistical difference obtained between fresh or vitrified-thawed embryo-transfer in ICSI cycles as regard pregnancy rate and pregnancy outcomes, which encourages freeze all strategy in low resources countries. Which.

\section{CONFLICT OF INTEREST}

There are no conflicts of interest.

\section{ACKNOWLEDGEMENT}

We have not received any funding from any corporate body or pharmaceutical company.

\section{REFERENCES}

1. Basirat Z., Rad, HA, Esmailzadeh S, Jorsaraei SG, Hajian-Tilaki K, Pasha H., et al. Comparison of pregnancy rate between fresh embryo transfers and frozen-thawed embryo transfers following ICSI treatment Int J Reprod Biomed (Yazd). 2016; 14(1): $39-46$

2. Al-Ghamdi A, Coskun S, Al-Hassan S, Al-Rejjal R, Awartani K. The correlation between endometrial thickness and outcome of in vitro fertilization and embryo transfer (IVF-ET) outcome. Reprod Biol Endocrinol 2008; 6: Article No. 37.

3. Aflatoonian A, Moghaddam FM, Mashayekhy M, Mohamadian F. Comparison of early pregnancy and neonatal outcomes after frozen and fresh embryo transfer in ART cycles J Assist Reprod Genet 2010; 27(12): 695-700
4. Henningsen AK, Pinborg A, Lidegaard Ø, Vestergaard C, Forman JL, Andersen AN. Perinatal outcome of singleton siblings born after assisted reproductive technology and spontaneous conception: Danish national sibling-cohort study. Fertil Steril 2011; 95(3): 959-963.

5. Roque M, Lattes K, Serra S, Solà I, Geber S, Carreras $\mathrm{R}$, et al. Fresh embryo transfer versus frozen embryo transfer in in vitro fertilization cycles: A systematic review and meta-analysis. Fertil Steril 2013; 99(1): 156-162.

6. 6- Pelkonen S, Koivunen R, Gissler M, NuojuaHuttunen S, Suikkari AM, Hyden-Granskog C, et al. Perinatal outcome of children born after frozen and fresh embryo transfer: The Finnish cohort study 1995 2006. Hum Reprod 2010; 25(4): 914-923.

7. Speroof L, Fritz MA. Clinical gynecologic endocrinology and infertility. 8th Ed. Philadelphia: Lippincott Williams \& Wilkins; 2011.

8. Aubuchon M, Burney RO, Schust DJ, Yao MWM, Berek J.S. Berek \& Novak Gynecology. 15th Ed. lippincott Williams \& Wilkins; 2012. Infertility and assisted Reproductive technology.

9. Belva F, Henriet S, Van den Abbeel E, Camus M, Devroey P, Van der Elst J, et al. Neonatal outcome of 937 children born after transfer of cryopreserved embryos obtained by ICSI and IVF and comparison with outcome data of fresh ICSI and IVF cycles. Hum Reprod 2008; 23(10): 2227-2238.

10. Westergaard HB, Johansen AM, Erb K, Andersen AN Danish National In-Vitro Fertilization Registry 1994 and 1995: A controlled study of births, malformations and cytogenetic findings. Hum Reprod 1999; 4(7): 1896-1902.

11. Oron G, Son WY, Buckett W, Tulandi T, Holzer H. The association between embryo quality and perinatal outcome of singletons born after single embryo transfers: A pilot study. Hum Reprod 2014; 29(7): 1444- 1451.

12. Glujovsky D, Pesce R, Fiszbajn G, Sueldo C, Hart RJ, Ciapponi A. Endometrial preparation for women undergoing embryo transfer with frozen embryos or embryos derived from donor oocytes. Cochrane Database Syst Rev 2010; 20: CD006359.

13. Zhou F, Lin XN, Tong XM, Li C, Liu L, Jin XY, et al, A frozen-thawed embryo transfer program improves the embryo utilization rate. Chin Med J (Engl) 2009; 122(17): 1974 -1978. 
14. AbdelHafez FF, Desai N, Abou-Setta AM, Falcone T, Goldfarb J. Slow freezing, vitrification and ultrarapid freezing of human embryos: A systematic review and metaanalysis. Reprod Biomed Online 2010;20: 209-222.

15. Fitzmaurice j ,boylan c, mcclure n. Are pregnancy rates compromised following embryo freezing to prevent OHSS?. Ulster Med J 2008; 77(3): $164-167$

16. Check JH, choe JK, nazari A, fox F, Swenson K. Fresh embryo transfer is more effective than frozen for donors oocyte recipients but not for donors. Hum reprod 2001; 16(7): $1403-1408$.

17. Zhu D, Zhang J, Cao S, Zhang J, Heng BC, Huang $\mathrm{M}$, et al. Vitrified-warmed blastocyst transfer cycles yield higher pregnancy and implantation rates compared with fresh blastocyst transfer cycles-time for a new embryo transfer strategy? Fertil Steril 2011; 95(5): 1691-1695.

18. Salumets A, Suikkari AM, Mäkinen S, Karro H, Roos A, Tuuri T. Parameters guiding selection of best embryos for transfer after cryopreservation: A reappraisal. Hum Reprod 2006 ; 21(9):2368- 2374.

19. Achache H, Revel A. Endometrial receptivity markers, the journey to successful embryo implantation. Hum Reprod Update 2006; 12(6): 731-746.

20. Guerif F, Bidault R, Cadoret V, Couet ML, Lansac $\mathrm{J}$, Royere D. Parameters guiding selection of best embryos for transfer after cryopreservation: A reappraisal. Hum Reprod 2002;17(5): 1321-1326. 